\title{
The Performance Diagnostic Checklist-Human Services: a Correction
}

\author{
James E. Carr ${ }^{1}$ • David A. Wilder ${ }^{2}$
}

Published online: 3 November 2015

(C) Association for Behavior Analysis International 2015

\begin{abstract}
The Performance Diagnostic Checklist (PDC) has been used in a number of investigations to assess the environmental determinants of substandard employee performance. Carr et al. (2013) revised the PDC to explicitly assess the performance of employees in human-service settings who are responsible for providing care to others: the Performance Diagnostic Checklist-Human Services (PDC-HS). The originally published PDC-HS contained three minor scoring errors, which have been corrected in the present version.
\end{abstract}

Keywords Functional assessment · Performance assessment . Performance diagnostic checklist · Performance management . Staff management

\section{Implications for Practice}

1. The literature on performance diagnostics suggests that identifying the reasons why employee performance is substandard—aka, a functional assessment—can lead to effective intervention.

2. The Performance Diagnostic Checklist-Human Services (PDC-HS) is an assessment for human-services staff that

Electronic supplementary material The online version of this article (doi:10.1007/s40617-015-0099-3) contains supplementary material, which is available to authorized users.

David A. Wilder

dawilder@fit.edu

1 Behavior Analyst Certification Board, Littleton, CO, USA

2 School of Behavior Analysis, Florida Institute of Technology, $150 \mathrm{~W}$. University Blvd., Melbourne, FL 32901, USA can be used to identify potential causes of their problematic behavioral excesses or deficits.

3. The main areas assessed by the PDC-HS are as follows: training, task clarification and prompting, resources, materials, and processes, and performance consequences, effort, and competition.

4. The PDC-HS concludes with an intervention planner that matches scored assessment items with sample interventions.

The Performance Diagnostic Checklist—Human Services assessment, originally published in Carr, Wilder, Majdalany, Mathisen, and Strain (2013), contained three minor scoring errors. An item scored as NO on the PDC-HS is suggestive of a potential problem and, thus, indicates an opportunity for a specific intervention to improve staff performance. However, item 5 in the resources, materials, and processes section and items 4 and 5 in the performance consequences, effort, and competition section are worded such that an answer of YES (instead of NO) is suggestive of a potential problem. These three items have been reworded in the accompanying assessment such that all PDC-HS items are aligned, with answers of NO being suggestive of a potential problem.

The online version of the original article can be found at http://www.ncbi.nlm.nih.gov/pmc/articles/PMC3680147

\section{Reference}

Carr, J. E., Wilder, D. A., Majdalany, L., Mathisen, D., \& Strain, L. A. (2013). An assessment-based solution to a human-service employee performance problem: an initial evaluation of the Performance Diagnostic Checklist-Human Services. Behavior Analysis in Practice, 6(1), 16-32. 